\title{
High Thoracic Epidural Anesthesia in Cardiac Surgery as a Sole Anesthetic Technique. Series of 12 Interventions.
}

\author{
Gentian Huti ${ }^{1}$, Asead Abdyli ${ }^{1}$, Rudin Domi ${ }^{1}$, Blerim Arapi ${ }^{1}$, Arben \\ Kojqiqi $^{2}$, Artan Jahollari ${ }^{2}$
}

https://doi.org/10.32391/ajtes.v3i2.50

\begin{abstract}
Epidural anesthesia has been used widely as a combination to general anesthesia in cardiac surgery since early seventies. The addition of this technique to general anesthesia may have beneficial effects on clinical outcome. Several clinical trials and experiments have shown that there may be significant benefits using epidural anesthesia in cardiac surgery. Somehow neuroaxial blockade manages to attenuate the response to surgical stress and improve perioperative analgesia. Between august 2014 and September 2018, high thoracic epidural anesthesia was applied to 12 patients as a sole anesthetic technique. Epidural anesthesia was chosen because the patients had relative contraindications to general anesthesia and mechanical ventilation. This paper gives an overview of epidural anesthesia usage and our experience.

Keywords: epidural anesthesia, cardiac surgery, perioperative analgesia.
\end{abstract}

*Corresponding Author: Gentian Huti

$=$ E-mail: gentianhuti@yahoo.com

${ }^{1}$ Department of Anesthesia and Intensive Care, American Hospital Tirana.

${ }^{2}$ Department of Cardiothoracic Surgery, American Hospital Tirana. 


\section{Full Text}

\section{Introduction}

Epidural anesthesia has been used widely as a combination to general anesthesia in cardiac surgery since early seventies. However its use as a sole technique in cardiac surgery dates long after. One of the first big performed a series of 137 patients with epidural anesthesia demonstrating its beneficial effects when used in selected patients (1). The addition of this technique to general anesthesia may have beneficial effects on clinical outcome (2). Several clinical trials and experiments have shown that there may be significant benefits using epidural anesthesia in cardiac surgery. Somehow neuroaxial blockade manages to attenuate the response to surgical stress and improve perioperative analgesia (3). After the introduction of off-pump technique another milestone in cardiac surgery was the use of high thoracic epidural anesthesia which made it possible to have more beneficial results in long-term management of postoperative pain in cardiac surgery. It made it possible to avoid the drawbacks of mechanical ventilation and general anesthesia in selected patients (4). Of course several studies have shown the high satisfaction rate of pain control in patients operated under high thoracic epidural anesthesia (5). Also the fact that on-pump surgery is done in normothermic temperatures, appears to give a more physiological approach to perioperative management of the patients (6).

\section{Methods and materials}

Between august 2014 and September 2018, after explaining and taking the permission from the patients, high thoracic epidural anesthesia was applied to 12 patients as a sole anesthetic technique, 8 male and 4 female. The catheter was inserted 24 hours prior to surgery at T3-T4 level in the first 8 cases. In the late 4 cases the catheter was inserted 1 hour before the surgery. Sensory block was tested with $2 \mathrm{ml}$ of lidocaine 2\%. Epidural anesthesia was chosen because the patients had relative contraindications to general anesthesia and mechanical ventilation. Their age varied from 52 to 81 years old. The most important and common characteristic of these patients was the poor pulmonary function test. Seven of them had mixed dysventilatory syndrome with no reversibility to any treatment. FEV1 ranged from 49-53\%. Anesthesia was obtained by infusion of bupivacaine $0.15 \mathrm{mg} / \mathrm{cm}$ and sulfentanyl $100 \mathrm{\gamma}$ initial dose and then continuous infusion of bupivacaine from epidural catheter with a dose of $0.06 \mathrm{mg} / \mathrm{cm} / \mathrm{h}$.

\section{Results}

Five patients had valve replacement surgery and seven CABG. One patient did off-pump CABG and the other six on-pump CABG. Three patients out of 12 had to go on pump twice due to hemorrhage. Two of them were put on pump immediately, intraoperatively, after the first time and the third one was re-operated 4 hours later due to blood 
loss. The patients were under epidural anesthesia and did not need to be intubated. Patients were followed up thirty days after the operation. No mortality was observed 3 months postoperatively. Life threatening arrhythmia was seen in only one patient who was intubated during the perioperative period. He had on -pump 3-vessel CABG. He had dilated cardiomyopathy with an EF of $30 \%$. The operation was successful without any intra-operatory complication. Immediately after being transferred to ICU the patient had a cardiac arrest. He was intubated urgently and cardiopulmonary assistance was given. He managed to recover despite a long stay in ICU. He was transferred to the ward 7 days after surgery and left hospital two weeks after in a very good condition. Ten patients did not need ICU assistance more than the day of the operation. They were transferred to the ward the second day and 9 of them were discharged without any complication the fifth day after the surgery. One patient left hospital at the seventh day. One patient was transferred to the ward immediately after the operation. She was mobilized 1 hour after the surgery and did not experience any complication nor feel any pain.

\section{Conclusion}

We can definitely say that epidural anesthesia is a very suitable technique when it comes to perioperative pain management in patients undergoing cardiac surgery. The postoperative management of these patients is easier due to the low incidence of complications and the early mobilization. Generally no life threating arrhythmias are seen in comparison to their incidence in patients operated under general anesthesia. Studies show that there is a low probability of epidural hematoma, varying approximately 1:12 000 to 1:150000 which is comparable to noncardiac surgery (7). Epidural anesthesia is a very suitable technique in selected patients with contraindications to general anesthesia and mechanical ventilation. However more studies and experience is needed to have a better view of the benefits, indications and uses of this technique in cardiac surgery.

\section{References}

1. J Thorac Cardiovasc Surg. 2003 Jun;125(6):1401-4.

Coronary artery bypass grafting in the awake patient: three years' experience in 137 patients. Karagoz HY1, Kurtoglu M, Bakkaloglu B, Sonmez B, Cetintas T, Bayazit K.

2. Anesthesiology. 2011

Feb;114(2):262-70. doi: 10.1097/ALN.0b013e318201d2de. Thoracic epidural anesthesia for cardiac surgery: a randomized trial. Svircevic V1, Nierich AP, Moons KG, Diephuis JC, Ennema JJ, Brandon Bravo Bruinsma GJ, Kalkman CJ, van Dijk D.

3. Tex Heart Inst J. 2006; 33(2): 148153. PMCID: PMC1524708 High Thoracic Epidural Anesthesia in Cardiac Surgery. Risk Factors for Arterial Hypotension. Stefano Casalino, MD, Fabio Mangia, MD, Edmond Stelian, MD, Eugenio Novelli, PhD, Marco Diena, MD, and Ugo F. Tesler, MD 
4. J Thorac Cardiovasc Surg. 2003 Jun;125(6):1394-400. Operative techniques in awake coronary artery bypass grafting. Aybek T1, Kessler P, Khan MF, Dogan S, Neidhart G, Moritz A, WimmerGreinecker G.

5. Med Arh. 2012; 66(5): 340-343 doi: 10.5455/medarh.2012.66.340-343.

Benefits, Risks and Complications of Perioperative Use of Epidural Anesthesia. Dragana UnicStojanovic, Srdjan Babic, Miomir Jovic.

6. Gaillard D, Bical O, Paumier D, Trivin F. A review of myocardial normothermia: its theoretical basis and the potential clinical benefits in cardiac surgery. Cardiovasc Surg 2000;8:198-203.

7. Curr Opin Anaesthesiol. 2009 Feb;22(1):84-7. doi: 10.1097/ACO.0b013e32831a40b6. High thoracic epidural anaesthesia for cardiac surgery. Royse CF1. 